\title{
MOTIVOS DE ESTUDIANTES DE NUEVO INGRESO PARA ESTUDIAR UN POSGRADO EN EDUCACIÓN ${ }^{1}$
}

\author{
Landy A. Esquivel Alcocer y Cecilia A. Rojas Cáceres \\ Universidad Autónoma de Yucatán, México
}

\section{INTRODUCCIÓN}

La Secretaría de Educación Pública (SEP) informa que, de 1990 a la fecha, se observa un crecimiento en la matrícula de la educación superior de más de 46 por ciento, cifra que representa el doble de la matricula superior atendida en $1970 \mathrm{y}$, atribuye este incremento: 1) al resultado del propio crecimiento demográfico, 2) a la mayor cobertura de la educación básica y la media superior, 3) al interés creciente de las personas por mejorar sus niveles de preparación, 4) a los esfuerzos realizados por los gobiernos federal y estatales y por las instituciones particulares para ampliar la oferta de opciones educativas para los egresados de la educación media superior. De acuerdo con la SEP, el nivel con más dinamismo en este incremento de matrícula es el posgrado, ya que en 1999 la matrícula se incrementó un 167\% y se atendía a 77 mil estudiantes más que en 1990. Este crecimiento de la matrícula es resultado tanto del interés de la población mexicana por alcanzar niveles más elevados de instrucción como del apoyo otorgado a los profesores de educación superior para que completen o amplíen sus estudios de posgrado. (Perfil de la Educación en México, 1999: 75-76).

¿Por qué se ha incrementado el interés de la población mexicana por alcanzar niveles de educación más elevados? Las razones pueden ser muy diversas, pero la psicología intenta explicar el por qué la gente se comporta como lo hace, a través de las teorías de la motivación.

\section{MARCO TEÓRICO}

\section{La motivación: un constructo psicológico}

La motivación se define como "aquello que energiza y dirige la conducta" (Woolfolk, 1996: 326), o como "un constructo hipotético usado para explicar el inicio, dirección, intensidad y persistencia de la conducta dirigida hacia un objetivo" (Good y Brophy, 1996: 295). Debido a la complejidad de definir el término motivación, se ha generado una diversidad de teorías conductistas, humanistas y cognoscitivas que varían en el grado en que se enfocan en factores biológicos, conductuales, cognitivos o sociales, pero que en lo fundamental, se proponen explicar la energía que guía el comportamiento de las personas en una determinada dirección. En particular, este estudio, se enmarcó en los enfoques humanistas y cognoscitivos de la motivación basados en las teorías de las necesidades.

Los teóricos humanistas creen que las personas actúan sobre sus ambientes y hacen elecciones respecto de qué hacer, pero están más interesadas en el desarrollo personal, la realización del potencial y la eliminación de obstáculos para la maduración personal. Los psicólogos cognoscitivistas, por otra parte, creen que los procesos de pensamiento controlan la conducta y se enfocan en la manera como las personas procesan la información e interpretan los significados personales en situaciones particulares. Entre 
las teorías iniciales que explican la motivación se encuentran las teorías de las necesidades que incluyen elementos cognoscitivos y humanistas, y para fines de este estudio se seleccionaron las siguientes:

\section{Teoría de necesidades de H. Murray}

Henry Murray define a la necesidad como una fuerza que influye en la percepción y el comportamiento de las personas por cambiar una situación insatisfactoria, esta necesidad genera una tensión que conduce a la persecución de un objetivo, el cual si es logrado liberará la tensión sentida y restaurará el equilibrio en la persona. De acuerdo con la teoría de Murray, existen 20 necesidades sociales aprendidas. Para la presente investigación se eligieron las siguientes: necesidad de logro; necesidad de afiliación, formar amistades y asociaciones, necesidad de poder o dominancia, controlar a los demás y necesidad de comprensión, analizar la experiencia (Good y Brophy, 1996: 297-299).

\section{Teoría de las necesidades de A. Maslow}

La jerarquía de necesidades humanas se clasifica a su vez en necesidades por deficiencia y necesidades de ser. Las necesidades por deficiencia son cuatro: necesidades fisiológicas (hambre, sueño, sed, sexo y descanso), necesidades de seguridad (protección, orden, estabilidad), necesidades de afiliación (afecto, aceptación social, amor, pertenencia), necesidades de autoestima (reconocimiento, respeto, prestigio, reputación, posición social). Las necesidades del ser son: necesidad de logros intelectuales, entendimiento, exploración, necesidad de apreciación estética, orden, estructura y belleza, necesidades de autorrealización (realización personal o llegar a ser lo que uno quiere ser, logros personales) (Woolfolk, 1996:333). En este estudio se eligieron las siguientes necesidades: seguridad, afiliación, autoestima, logros intelectuales y autorrealización.

\section{Teoría de la motivación de logro de D. McClelland y R. Atkinson}

De acuerdo con los teóricos de la necesidad de logro, la conducta es producto de tres factores: 1) la necesidad de tener éxito, 2) la probabilidad de éxito y 3) la necesidad de evitar el fracaso; y que la fuerza de la motivación de una persona para el logro de un objetivo particular está determinada por las fuerzas relativas de la motivación para enfocar la tarea y tratar de tener éxito en ella y evitar el fracaso. Para este estudio se eligió dentro de la necesidad de logro el factor tener éxito como una de las necesidades de los estudiantes para estudiar el posgrado.

\section{Teoría de la motivación hacia la estimulación}

Esta teoría sostiene que algunos seres humanos están constantemente en la búsqueda de estímulos nuevos, tratan de conservar determinados niveles de estimulación y actividad óptimas, aumentándolos o reduciéndolos, según sea necesario. El nivel óptimo de excitación deseado varía de individuo a individuo y algunas personas intentan evitar el aburrimiento buscando situaciones desafiantes que impliquen un cambio en sus vidas (Feldman, 1998: 295; Lahey, 1999: 420). La motivación hacia la estimulación está representada en este estudio por la motivación de cambio.

En conclusión, se puede decir que, de acuerdo con estas teorías, si un estudiante tiene una necesidad (psicológica o social) ésta le origina un estado de tensión que necesita mitigar. Para disminuir esta tensión realiza una acción (se inscribe en el programa) y desarrolla un esfuerzo que se traduce en conductas (asistir a clases, trabajar en grupo, cumplir con las tareas, etc.) porque tiene la expectativa que 
este esfuerzo le permitirá alcanzar su meta. Si su esfuerzo es recompensado con el logro de su meta, entonces su necesidad será satisfecha y disminuirá la tensión.

\section{La motivación como factor interno asociado al éxito en los estudios}

Sánchez y Valdés (2003) mencionan tres grandes factores asociados con el desempeño del estudiante: 1) los individuales, como el intelecto, los motivos, las emociones y los aspectos sociales; 2) los factores asociados con la escuela, como el cuerpo docente, los programas, etc; y 3) los factores asociados con la familia, como son la posición socioeconómica, el nivel educativo de los padres, las expectativas de estos respecto de la educación de sus hijos, entre otros.

De igual manera, Esquivel (2002), en su modelo conceptual de los factores que influyen en el desempeño escolar, se refiere a los factores internos como aquellos atribuidos al mismo estudiante y que lo caracterizan o definen (su capital cultural, sus características físicas, morales, cognitivas, personales, su locus de control, su motivación, sus atribuciones causales, sus hábitos de estudio, etc.), y a los factores externos como los sistemas sociales es decir, la familia (tipo y tamaño, ambiente familiar, características demográficas, nivel educativo, social y económico de los padres y los valores familiares, entre otros), la escuela (tipo de escuela, condiciones físicas y ambientales, profesores, asignaturas, etc.), y otros sistemas sociales como la iglesia, el grupo de coetáneos y los medios de comunicación, que influyen en el comportamiento en la escuela. El punto medular de este modelo es que ambos factores (tanto internos o externos) no sólo están asociados con el desempeño escolar, sino que pueden ser los mismo factores asociados tanto al éxito escolar, como al fracaso escolar, de tal manera que es la complejidad de estas combinaciones la que determina la dirección que pueda tener esta relación.

Es así como Esquivel (2002) y Sánchez y Valdés (2003) incluyen en sus modelos a la motivación como uno de los factores internos más importantes en el contexto educativo. Después de todo, los motivos han sido mencionados por los psicólogos educativos como uno de los constructos psicológicos íntimamente relacionados con el desempeño y el éxito escolar (Arancibia, Herrera, y Strasser,1999; Brophy, 1997; Sprinthall, Sprinthall y Oja 1996, y Woolfolk, 1996).

\section{La motivación en los estudiantes del posgrado en educación}

En relación con las motivaciones de los estudiantes para inscribirse en el posgrado, Arredondo (2000: 144) menciona las siguientes: la formación personal, la obtención de un grado académico, las mayores posibilidades de empleo y de mejor remuneración y el prestigio de la institución. Tres de las motivaciones se pueden clasificar como extrínsecas y relacionadas con las necesidades de seguridad, reconocimiento y autorrealización. En ninguno de los casos se menciona la necesidad de conocimiento o de logro intelectual que sería una de las necesidades intrínsecas de orden superior en la jerarquía de Maslow y posiblemente la más importante para el logro y éxito en los estudios de posgrado.

Por su parte, Soria (2000: 28) menciona que los motivos de la demanda de posgrado son entre otros: la certificación, progreso en el escalafón, el aumento salarial (a los que considera como satisfactores humanos básicos), la carrera universitaria (PROMEP y SUPERA), los ascensos en la estructura de poder hacia la administración central, el desarrollo profesional, la oportunidad vocacional tardía, el desarrollo personal y el desempleo. De nuevo encontramos que es la motivación extrínseca y no intrínseca la que mueve a los estudiantes hacia el posgrado y que los alumnos no mencionan la necesidad de conocimiento o de logro intelectual. 
En la literatura se reportan otros estudios acerca de la motivación de los estudiantes para ingresar a una carrera universitaria. Por ejemplo, en el estudio realizado por Barnetson (1997) con una muestra de 77 estudiantes de la Universidad de Calgary, Canadá, se utilizó un cuestionario de información biográfica que incluía preguntas acerca de las razones para asistir a dicha universidad. Entre los motivos más frecuentemente expuestos por los estudiantes se encontraron: lograr un nivel estándar más alto de vida, la ampliación de las oportunidades de obtener un empleo, el deseo de obtener un grado, pasarla bien, llegar a ser autosuficiente, aprender nuevas cosas, aumentar sus conocimientos, conocer nuevas personas y la calidad de la enseñanza en dicha universidad. Cinco de estos motivos son extrínsecos; sin embargo, aparecen dos motivos relacionados con la necesidad de conocimiento que no aparecieron en los dos estudios con estudiantes mexicanos.

Otro estudio es el llevado a cabo por Papanastasiou y Papanastasiou (1997) para conocer los factores que influyen en los estudiantes para querer ser profesores (176 estudiantes de la Universidad de Cyprus y 157 estudiantes de la Universidad Estatal de Pennsylvania) encontraron que los motivos internos fueron los que influyeron más fuertemente en los estudiantes de la Universidad Estatal de Pennsylvania, mientras que los factores que influyeron más en los estudiantes de Cyprus fueron los beneficios y el estatus de la profesión.

En el estudio de Wu (2000) sobre los motivos expresados por las estudiantes de origen chino para elegir la carrera de periodismo se encontró que las razones que las estudiantes expusieron para elegir esta carrera fueron: por una motivación profesional, para desarrollar su potencial y por el amor que experimentan hacia el arte de escribir, y no por el deseo de tener fama o por lograr un mejor estatus social, es decir, sus tres motivos fueron intrínsecos.

Por su parte, Gámez (2000) en su investigación de los motivos principales para elegir la carrera de Psicología, realizada en la Universidad de la Laguna, España, encontró que los motivos más relevantes fueron los motivos de superación de problemas personales y preocupación por las demás personas, mismos que pueden clasificarse como motivos internos.

En la investigación realizada por Ramos y Esquivel (2002), de motivos hacia la carrera de Psicología de la Universidad de Colima, realizada con mujeres solteras aspirantes a la licenciatura en Psicología en el ciclo escolar 2000-2001, se encontró que el motivo principal por el cual ingresan las estudiantes en la licenciatura es por autorrealización, es decir, por superación personal, seguido por motivos de seguridad, como obtener un trabajo y tener una mejor calidad de vida. En la investigación realizada por Esquivel y Arjona (2003) con estudiantes de nuevo ingreso en los posgrados en educación (Especialización en Docencia y Maestría en Educación Superior, ciclo escolar 2001-2002), también se encontró que los motivos más importantes expresados por los estudiantes son de carácter intrínseco, tales como la autorrealización y el conocimiento per se.

\section{JUSTIFICACIÓN DEL ESTUDIO}

Conocer los motivos que subyacen en el interés creciente de la población mexicana por mejorar sus niveles de preparación es muy importante, ya que en la actualidad, tanto los psicólogos como los educadores reconocen el relevante papel que juega la motivación en la conducta humana como un componente esencial del aprendizaje, así como en un factor asociado con el rendimiento, permanencia y 
egreso de los estudiantes de la escuela y en general, en el éxito académico (Sprinthall, Sprinthall y Oja,1996: 416), independientemente del programa de estudios que se curse.

\section{OBJETIVO DE LA INVESTIGACIÓN}

Jerarquizar los motivos de los estudiantes de nuevo ingreso para estudiar los programas de la Especialización en Docencia, la Maestría en Investigación Educativa y la Maestría en Administración de Organizaciones Educativas de la Facultad de Educación de la Universidad Autónoma de Yucatán en el ciclo escolar 2004-2005.

\section{PREGUNTA DE INVESTIGACIÓN}

¿Cuáles son los motivos que expresan los estudiantes para inscribirse a los posgrados en educación que ofrece la Facultad de Educación de la Universidad Autónoma de Yucatán en el ciclo escolar 2004-2005?

\section{MÉTODO}

\section{Población}

La población estuvo conformada por 63 estudiantes: 18 estudiantes de la Especialización en Docencia, 13 de la Maestría en Investigación Educativa y 32 de la Maestría en Administración de Organizaciones Educativas. Dado el número de estudiantes se optó por censo en lugar de muestreo.

\section{Instrumentos}

Se utilizó el Cuestionario de Motivación hacia el Posgrado en Alumnos de Nuevo Ingreso a un Posgrado en Educación de Esquivel y Arjona $(2002)^{2}$, el cual se compone de una lista de 30 motivos para estudiar un posgrado, los cuales son jerarquizados por los estudiantes en una escala tipo Likert en las siguientes categorías: ningún valor (0), poco valor (1), regular valor (2), alto valor (3), muy alto valor (4). Se utilizó también una pregunta de respuesta abierta ${ }^{3}$ acerca de los motivos por los cuales desean estudiar el posgrado. La finalidad de esta pregunta fue validar el constructo de "motivación hacia el posgrado.

Las respuestas al cuestionario y a la pregunta abierta se clasificaron en ocho categorías de motivos: seguridad, afiliación, reconocimiento, logro, poder, conocimiento autorrealización, cambio y, que se definen a continuación:

1) seguridad (un aumento de salario, conseguir trabajo, sentir más confianza al dar clases y conservar el empleo) mencionada en la jerarquía de necesidades de Maslow, se refiere a la necesidad de las personas de sentirse protegidas tanto física, como psicológicamente y tener estabilidad económica;

2) afiliación o afecto (conocer a otras personas, ser apreciado por mis alumnos, conocer a otros colegas de la docencia y hacer nuevos amigos). Referida en las teorías de Maslow y de Murray como el deseo de las personas de formar amistades y asociaciones, de ser aceptadas o de pertenecer a un grupo;

3) reconocimiento (un mayor reconocimiento de mis colegas, mejorar mi autoestima, incrementar mi prestigio como profesor y mejor valoración de mi trabajo por parte de mis superiores), Esta 
categoría es tomada de la teoría de Maslow y se refiere a la necesidad de las personas de valorarse a sí mismas y al prestigio, reputación o posición social ante los demás;

4) logro (la obtención de un grado, tener más éxito en mi trabajo, superar nuevos retos profesionales y ascender en mi trabajo), es mencionada en las teorías de Murray y de McClelland como la necesidad de las personas de vencer los obstáculos y tener éxito en las tareas que realizan;

5) poder (obtener un cargo de representación de mis colegas, ser líder de opinión en algunos asuntos educativos, ocupar un cargo directivo en mi lugar de trabajo e influir más en mis compañeros de trabajo), de acuerdo con la teoría de Murray es el deseo de ejercer poder o dominar se refiere a la necesidad de controlar a los demás;

6) conocimiento (comprender más el fenómeno educativo, aprender cosas nuevas, mejorar mis técnicas para impartir clases o hacer investigación y una mejor capacitación general de mi trabajo, interés por conocer, comprender o estudiar), mencionada en la teoría de Murray (necesidad de comprensión) se refiere a analizar la experiencia, y en la teoría de Maslow la encontramos en la jerarquía de necesidades del ser refiriéndose a la aspiración del ser humano de tener logros intelectuales y a su apetito de conocimiento, entendimiento y exploración intelectual;

7) autorrealización (una superación personal, sentirme satisfecho con lo que hago, un sentimiento de autorrealización y sentirme autosuficiente como profesor), Está de acuerdo con la jerarquía de Maslow, que se refiere a la necesidad de la persona por superars e de manera permanente, por el placer mismo de su superación;

8) cambio (estimular mi desarrollo intelectual, cambiar de lugar de trabajo, preparación para cambiar de tipo de trabajo y escapar de la rutina diaria).

\section{Procedimiento}

Se administraron los cuestionarios a los aspirantes en el mes de julio de 2004 y los datos se capturaron en una base de datos en el programa SPSS versión 9.0. Para realizar el procesamiento de los mismos se utilizó el mismo programa. Los datos se analizaron utilizando las medidas de tendencia central (media, mediana, y moda) y la prueba no Paramétrica $W$ de Kendall. Esta es una medida que estima el grado acuerdo entre diferentes jueces o evaluadores y es interpretada como un coeficiente de concordancia entre los sujetos, que va de cero a uno, donde cero significa "ningún acuerdo" y uno "completamente de acuerdo", a partir de estos estadísticos se jerarquizaron los motivos, por programa y por género. Por último, se obtuvo un índice de correlación entre las categorías de motivos para determinar si es que eran mutuamente excluyentes o si guardaban relación entre ellas.

Las respuestas obtenidas en la pregunta abierta, sobre los motivos de los estudiantes para estudiar un posgrado en educación, se analizaron en términos de sus frecuencias y se clasificaron en ocho categorías de motivos: seguridad, afiliación, reconocimiento, poder, conocimiento, autorrealización, cambio y logro, y se jerarquizaron en función de sus frecuencias. Las respuestas al Cuestionario de Motivación hacia el Posgrado en Alumnos de Nuevo Ingreso a un Posgrado en Educación se analizaron y se hicieron las jerarquías de los motivos utilizando las medidas de tendencia central de media, mediana y moda. 
También se utilizó la prueba $\mathrm{W}$ de Kendall para jerarquizar los motivos de acuerdo con el grado de concordancia entre los estudiantes.

\section{RESULTADOS}

Programa Especialización en Docencia. (ED)

La jerarquía de los motivos expresados por los 18 aspirantes en la pregunta de respuesta abierta se muestra en la tabla 1. Como puede observarse, los primeros lugares de la jerarquía de motivos lo ocupan los motivos intrínsecos de autorrealización y conocimiento, seguido de los motivos extrínsecos de reconocimiento, poder y seguridad, quedando en último lugar los motivos de cambio logro y afiliación.

\section{TABLA 1}

Ordenamiento de motivos para estudiar el programa de especialización en docencia, de acuerdo con las frecuencias de las respuestas

\begin{tabular}{|l|l|}
\hline Categorías de motivos & Orden \\
\hline Autorrealización & 1 \\
Conocimiento & 2 \\
Reconocimiento & 3 \\
Poder & 4 \\
Seguridad & 4 \\
Cambio & 5 \\
Logro & 5 \\
Afiliación & 6 \\
\hline
\end{tabular}

Con la finalidad de verificar el ordenamiento de los motivos, se realizó el análisis de acuerdo con las medidas de tendencia central, media mediana y moda. Observando la tabla 2, podemos comprobar que el ordenamiento por frecuencias se confirma pues los motivos internos de autorrealización y conocimiento vuelven a aparecer en los primeros lugares, y los motivos externos más comunes como seguridad, reconocimiento y poder aparecen en los lugares subsecuentes.

\section{TABLA 2}

Comparación y ordenamiento de los motivos de acuerdo a las medidas tendencia central media, mediana y moda.

\begin{tabular}{|l|l|l|l|l|l|l|}
\hline & Media & Orden & Mediana & Orden & Moda & Orden \\
\hline Autorrealización & 18.06 & 1 & 18 & 1 & 20 & 1 \\
Conocimiento & 15.28 & 2 & 16 & 2 & 17 & 2 \\
Seguridad & 14 & 3 & 14.5 & 3 & 16 & 3 \\
Reconocimiento & 10.72 & 4 & 10.5 & 4 & 9 & 4 \\
Poder & 9.5 & 5 & 9 & 5 & 8 & 5 \\
Afiliación o afecto & 8.67 & 6 & 8.5 & 6 & 8 & 5 \\
Logro & 6.5 & 7 & 7 & 7 & 8 & 5 \\
Cambio & 6 & 8 & 7 & 7 & 7 & 6 \\
\hline
\end{tabular}

La tabla 3 muestra el orden de los motivos de acuerdo con el índice de concordancia entre los sujetos aspirantes de la ED por medio de la estadística no paramétrica $W$ de Kendall. Respecto al coeficiente de concordancia (.825) puede apreciarse que es significativamente alto pues se acerca a uno, es decir al completo acuerdo, lo cual confirma el orden de los motivos de los otros análisis realizados. 
TABLA 3

Ordenamiento de los motivos hacia la especialización en docencia, de acuerdo con los rangos promedios de la prueba Coeficiente de concordancia W de Kendall(.825) y (N=18)

\begin{tabular}{|l|l|l|}
\hline Categoría de motivos & Rango promedio & Ordenamiento de motivos \\
\hline Autorrealización & 7.94 & 1 \\
Conocimiento & 6.42 & 2 \\
Seguridad & 6.31 & 3 \\
Reconocimiento & 4.42 & 4 \\
Poder & 3.78 & 5 \\
Afiliación & 3.28 & 6 \\
Logro & 1.97 & 7 \\
Cambio & 1.89 & 8 \\
\hline
\end{tabular}

\section{Programa Maestría en Investigación Educativa (MIE)}

La tabla 4 presenta el ordenamiento de las categorías de motivos reportados por los aspirantes de la MIE en la pregunta de respuesta abierta, obtenido a partir de las frecuencias de los mismos, el cual muestra que nuevamente hubo correspondencia entre lo esperado y lo obtenido, pues los motivos con las mayores frecuencias se encuentran en la categoría de motivos internos y los motivos externos aparecen en los lugares subsecuentes.

\section{TABLA 4}

Ordenamiento de las categorías de motivos de acuerdo con las frecuencias (pregunta abierta).

\begin{tabular}{|l|l|}
\hline Categorías de motivos & Orden \\
\hline Autorrealización & 1 \\
Conocimiento & 2 \\
Reconocimiento & 2 \\
Cambio & 3 \\
Seguridad & 4 \\
Poder & 5 \\
Logro & 5 \\
Afiliación & 6 \\
\hline
\end{tabular}

Para comprobar este ordenamiento, en la tabla 5 se muestra el análisis comparativo de las medidas de tendencia central, media, mediana y moda. En esta tabla se puede observar una pequeña variación pues sólo los motivos de autorrealización se mantuvieron en el primer lugar, subieron un nivel los motivos de seguridad, se colocaron en tercer lugar los motivos de conocimiento, y los motivos externos de reconocimiento y poder se conservaron en el mismo orden

\section{TABLA 5}

Comparación de las categorías de motivos de acuerdo a las medidas de tendencia central media, mediana y moda.

\begin{tabular}{|l|l|l|l|l|l|l|}
\hline & Media & Orden & Mediana & Orden & Moda & Orden \\
\hline Autorrealización & 17.31 & 1 & 19 & 1 & 20 & 1 \\
Seguridad & 15.38 & 2 & 16 & 2 & 18 & 2 \\
Conocimiento & 14.54 & 3 & 15 & 3 & 16 & 3 \\
Reconocimiento & 11.23 & 4 & 11 & 4 & 11 & 4 \\
Poder & 10.85 & 5 & 11 & 4 & 8 & 6 \\
Afiliación & 8.54 & 6 & 8 & 5 & 8 & 6 \\
Cambio & 7.54 & 7 & 8 & 5 & 9 & 5 \\
Logro & 6.85 & 8 & 7 & 6 & 6 & 7 \\
\hline
\end{tabular}


La tabla 6 muestra el análisis de concordancia para verificar el orden obtenido, donde se puede apreciar que el orden resultante al aplicar la escala W de Kendall, se conserva con respecto al orden reportado por la media, y el índice de concordancia aparece un poco menor que el de la ED, pero considerablemente significativo en acuerdo, por lo tanto se mantiene y confirma el orden de los motivos.

\section{TABLA 6}

Ordenamiento de los motivos hacia la MIE, de acuerdo con los rangos promedios de la prueba Coeficiente de concordancia W de Kendall (.787). N=13

\begin{tabular}{|l|l|l|}
\hline Categoría de motivos & Rangos promedios & Ordenamiento \\
\hline Autorrealización & 7.35 & 1 \\
Seguridad & 6.85 & 2 \\
Conocimiento & 6.27 & 3 \\
Reconocimiento & 4.54 & 4 \\
Poder & 4.27 & 5 \\
Afecto & 2.73 & 6 \\
Cambio & 2.19 & 7 \\
Logro & 1.81 & 8 \\
\hline
\end{tabular}

\section{Maestría en Administración de Organizaciones Educativas (MAOE)}

En la tabla 7 se muestra el ordenamiento, de acuerdo con las frecuencias, de los motivos reportados por los aspirantes de la MAOE, en la pregunta abierta. Se puede observar franca consistencia con los resultados de los otros programas pues los motivos con las mayores frecuencias siguen estando en la categoría de motivos internos y los motivos externos aparecen en los lugares subsecuentes.

TABLA 7

Ordenamiento de las categorías de motivos de acuerdo con las frecuencias

\begin{tabular}{|l|l|}
\hline Categorías de motivos & Orden \\
\hline Autorrealización & 1 \\
Conocimiento & 2 \\
Reconocimiento & 3 \\
Cambio & 4 \\
Seguridad & 4 \\
Afiliación & 5 \\
Logro & 6 \\
Poder & 6 \\
\hline
\end{tabular}

La tabla 8 muestra el orden del análisis comparativo de las medidas de tendencia central, media, mediana y moda. En estas comparaciones se observa que los motivos externos aparecen desde el tercer puesto. Sin embargo los motivos internos de autorrealización y conocimiento siguen apareciendo en los primeros lugares. Comparando los resultados de la MAOE con los resultados de la ED, el ordenamiento es idéntico, pero en relación con la MIE hay una pequeña variación del orden de los motivos pues los motivos externos figuran desde el segundo puesto. 
TABLA 8

Comparación de las categorías de motivos de acuerdo a las medidas de tendencia central media, mediana y moda

\begin{tabular}{|l|l|l|l|l|l|l|}
\hline & Media & Orden & Mediana & Orden & Moda & Orden \\
\hline Autorrealización & 17.03 & 1 & 18 & 1 & 20 & 1 \\
Conocimiento & 13.56 & 2 & 14 & 2 & 16 & 2 \\
Seguridad & 12.41 & 3 & 12 & 3 & 14 & 3 \\
Poder & 11.78 & 4 & 12 & 3 & 12 & 4 \\
Reconocimiento & 10.09 & 5 & 10 & 4 & 16 & 2 \\
Afecto & 7.59 & 6 & 8 & 5 & 9 & 5 \\
Logro & 7.03 & 7 & 7 & 6 & 8 & 6 \\
cambio & 6.41 & 8 & 6.5 & 7 & 6 & 7 \\
\hline
\end{tabular}

El análisis de concordancia de Kendall se muestra en la tabla 9 que confirma el orden de la media y la mediana pues el índice de concordancia es muy significativo, lo cual quiere decir que los motivos internos de autorrealización y conocimiento se conservan en primer y segundo lugar, seguidos por los motivos externos de seguridad, poder y reconocimiento.

TABLA 9

Ordenamiento de los motivos hacia la MAOE, de acuerdo con los rangos promedios de la prueba Coeficiente de concordancia W de Kendall ( .730). N=32

\begin{tabular}{|l|l|l|}
\hline Categoría de motivos & Rangos promedios & Ordenamiento \\
\hline Autorrealización & 7.59 & 1 \\
Conocimiento & 6.19 & 2 \\
Seguridad & 5.89 & 3 \\
Poder & 5.33 & 4 \\
Reconocimiento & 4.05 & 5 \\
Afecto & 2.59 & 6 \\
Logro & 2.23 & 7 \\
Cambio & 2.13 & 8 \\
\hline
\end{tabular}

Jerarquización de los motivos hacia el posgrado (ED, ME, MAOE)

Tomando en cuenta los resultados de cada uno de los programas de posgrado se obtuvo un ordenamiento sin distinción de programa, representado en la tabla 10.

TABLA 10

Ordenamiento de los motivos hacia los programas de posgrado, de acuerdo con los rangos promedios de la prueba Coeficiente de concordancia W de Kendall (.749) N=63

\begin{tabular}{|l|l|l|}
\hline Categoría de motivos & Rangos promedios & Ordenamiento \\
\hline Autorrealización & 7.64 & 1 \\
Conocimiento & 6.27 & 2 \\
Seguridad & 6.21 & 3 \\
Poder & 4.67 & 4 \\
Reconocimiento & 4.25 & 5 \\
Afecto & 2.82 & 6 \\
Logro & 2.07 & 7 \\
Cambio & 2.07 & 7 \\
\hline
\end{tabular}

Un último análisis fue el de la correlación entre los motivos que se hace con el objetivo de determinar hasta que punto están relacionados unos con otros. De acuerdo con estas correlaciones: los motivos de seguridad están altamente vinculados con los de reconocimiento, es decir, se esperaría que una 
persona que reporta motivos de reconocimiento también reportara motivos de seguridad y ambas mantienen relaciones de reciprocidad.

Los motivos de seguridad están vinculados también con los de logro y conocimiento, aunque cabe mencionar que respecto de estos últimos la correlación es muy baja, y no se aprecia ninguna relación con otros motivos, por lo que podemos pensar que el motivo de conocimiento es casi independiente de los otros, es decir, que la necesidad de conocimiento no necesariamente esta ligada con otras necesidades. Los motivos de afecto están igualmente vinculados con los de reconocimiento y estos últimos están también vinculados significativamente con los de poder. Los motivos de cambio están relacionados con los de logro y los de autorrealización con los de afecto. Estos resultados se presentan en la tabla 11.

\section{TABLA 11}

Índice de correlación entre las categorías de motivos.

\begin{tabular}{|l|l|}
\hline Categorías de motivos & índice de correlación \\
\hline Seguridad- Reconocimiento & .77 \\
Afecto- Reconocimiento & .66 \\
Logro-Seguridad & .65 \\
Poder- Reconocimiento & .65 \\
Conocimiento- seguridad & .45 \\
Autorrealización- afecto & .45 \\
Cambio- logro & .61 \\
\hline
\end{tabular}

\section{DISCUSIÓN DE RESULTADOS}

De acuerdo con los resultados de este estudio, los motivos principales por los que las personas se inscriben en los programas de posgrado en Educación que ofrece la Facultad de Educación de la Universidad Autónoma de Yucatán son: en primer lugar, los de tipo intrínseco, el motivo de autorrealización, es decir, los estudiantes al inscribirse en los programas de posgrado buscan su superación personal o simplemente tener un sentimiento de autorrealización; en segundo lugar, el motivo de conocimiento, como adquirir nuevas estrategias de enseñanza o conocer más sobre el fenómeno educativo, aprender cosas nuevas y tener una mejor capacitación para el trabajo. Estos dos motivos, de autorrealización y de conocimiento quedan en la categoría de motivos del ser de acuerdo con la teoría de necesidades de Maslow y que se logran una vez cubiertas las necesidades por suficiencia. El que los dos principales motivos para estudiar el posgrado sean intrínsecos concuerda con los resultados obtenidos por Esquivel y Arjona (2003) en poblaciones de estudiantes de los programas de Especialización en Docencia y Maestría en Educación Superior en el ciclo escolar 2001-2002, quienes también encontraron que los motivos internos obtuvieron los primeros lugares, quedando en primer lugar el motivo de conocimiento.

En tercer lugar quedaron los motivos de seguridad cómo obtener o conservar el empleo, aspirar a un mejor sueldo o nivel de vida; lo que también es reportado en trabajos previos por Arredondo (2000) cuando dice que los motivos más comunes por los que los académicos se inscriben en el posgrado son de orden externo, entre ellos conservar el empleo u obtener un mejor nivel de vida. En la actualidad se sabe que el lograr un mejor trabajo y un salario más alto está en función de la preparación que el aspirante al puesto tenga. Es lógico esperar que una institución educativa con programas educativos de calidad tenga en su planta académica a profesores con niveles cuando menos de maestría. El mismo Programa de Mejoramiento del Personal de las universidades tiene como una de sus estrategias el reconocimiento del 
perfil del profesor de educación superior en el grado preferente o mínimo. También hay acuerdo con los resultados encontrados por Ramos y Esquivel (2002) quienes encontraron que las mujeres ingresan en una licenciatura por los motivos de autorrealización y superación personal, seguidos por los motivos de seguridad, como obtener un trabajo y tener una mejor calidad de vida. En el cuarto lugar quedaron los motivos de poder, como influir más en los compañeros de trabajo, ocupar un cargo de representación, ser líder de opinión en asuntos educativos, ocupar puestos directivos, etc. Este motivo también fue encontrado por Soria (2000), los profesores buscan ascender en el escalafón de la estructura organizacional no únicamente por el salario devengado sino también porque el puesto implica el ejercicio del poder en la institución.

Los motivos de reconocimiento aparecen en la posición cinco para los que buscan ser más apreciados por sus familiares, alumnos, compañeros o superiores, e incrementar su prestigio como profesionales de la educación. La necesidad de reconocimiento está clasificada por Maslow como una necesidad por deficiencia y está relacionada con la autoestima y la valía de la persona. Algunos de los estudiantes creen que el grado incrementará su prestigio en la comunidad en la que se desenvuelven. En sexto lugar quedaron los motivos de afiliación o de afecto, que de acuerdo con Maslow también pertenecen a la categoría de las necesidades por deficiencia. De acuerdo con los resultados de este estudio algunos estudiantes inician sus estudios de posgrado porque tienen la expectativa de conocer a otras personas, hacer amigos o encontrar personas con quienes compartir sus afectos. En séptimo lugar quedaron los motivos de logro, que según Murray y McClelland caracterizan a las personas que tienen la necesidad de vencer los obstáculos y tener éxito en las tareas que realizan y en octavo y último lugar quedaron los motivos de cambio que reflejan a los estudiantes que buscan cambiar de actividad o algo que los estimule o motive a llevar a cabo una nueva tarea en su vida profesional o personal.

Las comparaciones de las jerarquías entre los tres programas de posgrado demostraron que aunque hubo pequeñas variaciones en el orden, los motivos intrínsecos se mantuvieron en los primeros lugares de las tablas, independientemente del programa de estudios (especialización o maestría). Se hace notar también que de las correlaciones entre las categorías de motivos, destaca la correlación entre seguridad y reconocimiento (.77), al parecer ser reconocido por estudiar un posgrado de alguna manera incrementa la seguridad en sí mismo o la autoestima de la persona.

En conclusión, el iniciar una acción (inscribirse en un posgrado) puede ser resultado de diferentes motivos, ya sean estos expresados por una necesidad del ser o por una necesidad de deficiencia. De entre la jerarquía elegida de ocho motivos, destacan los motivos intrínsecos y entre éstos el motivo de conocimiento que se espera manifiesta un estudiante que además costea sus estudios por sus propios medios. Aunque no se encontraron diferencias en razón del programa de estudios, valdría la pena investigar si existen diferencias entre los motivos expresados por estudiantes que se encuentran en situaciones diferentes de las aquí expresadas, sobre todo entre aquellos que estudian costeándose a sí mismos sus estudios y aquellos que tienen una beca, o si existen diferencias por área del conocimiento, por género, por edad y si estos motivos permanecen durante el tiempo que dura el plan de estudios; o si existe una relación de los motivos con el rendimiento académico del estudiante en el programa y con su permanencia en el mismo. 


\section{NOTAS}

1. Esta investigación fue realizada en el marco del III Verano de la Investigación Científica de la Península de Yucatán 2004 , Organizado por la Universidad Autónoma de Yucatán como parte del programa de Impulso y Orientación a la Investigación (PRIORI).

2. El Cuestionario de Motivación hacia el Posgrado en Alumnos de Nuevo Ingreso a un Posgrado en Educación de Esquivel y Arjona (2002) se ha administrado en tres generaciones de alumnos al posgrado en la Facultad de Educación (2002, 2003 y 2004), como parte del proceso de admisión y selección a los programas de posgrado.

3. En el caso de la pregunta abierta, se realizó una validación por consenso y por criterio de la categorización de los motivos mencionados por los aspirantes, con la participación de tres maestros del área de posgrado de la facultad de educación y tres maestros del área de Licenciatura de la misma facultad.

\section{REFERENCIAS}

Arancibia, V. , HerRera, P. y Strasser, K. (1999). Psicología de la Educación. 2a Edición. Chile: Alfaomega-Universidad Católica de Chile.

ARREDONDO, G.(2000). La eficiencia de los Posgrados en Educación. Una perspectiva comparativa de los procesos de graduación en Moreno,B, Soria, N. Garibay, B. Sánchez, P.,Arredondo, G.,Sánchez, E. y Díaz, B.(2000). Problemática de los Posgrados en Educación en México: Hacia la consolidación en el siglo XXI. Universidad Autónoma del Carmen. Cuadernos de Investigación 5.

BARNETSON, R, J. (1997). Marketing the University of Calgary to Frosh: A Calgary._Canada.

EscAmiLlA, H. (1999) Determinantes del logro escolar en el nivel superior. Trabajo recepcional de Diplomado en Especialización en Orientación y Consejo Educativo. Mérida, Yucatán Facultad de Educación, Universidad Autónoma de Yucatán.

ESQUIVEL, L. (2002). Modelo conceptual de los factores que influyen en el desempeño escolar. En L. Esquivel, A. Valdés y H. Cervera. Sistema familiar de estudiantes con éxito y fracaso escolar. Ponencia presentada en Pedagogía 2003 Ciudad de la Habana, Cuba del 3 al 7 de febrero.

ESQUIVEL, L. y ARJONA, J. (2003) “Características demográficas, motivaciones y expectativas( laborales, personales y familiares) de estudiantes del posgrado de la facultad de Educación de la UADY". Proyecto de investigación. Unidad de Posgrado de Investigación de la Facultad de Educación de la Universidad Autónoma de Yucatán.

FEldman, R. S. (1998). Psicología con aplicaciones a los países de habla hispana. 3ª . Edición. McGrawHill: México.

GÁmeZ, E y MARRERO, H. (2000) Metas y motivos en la elección de la carrera de Psicología. Vol. 3 No. 5-6. [en red] Disponible en: http:/reme.uji.es

GoOD, T. L. y BROPHY, J. (1996). Psicología educativa contemporánea. 5a edición, McGraw Hill, México, INEGI (2001). SEP. Estadística Básica del Sistema Educativo Nacional. Inicio de cursos, 2000/2001

LAHEY, B.B. (1999). Introducción a la Psicología. México: McGraw-Hill

PAPANASTASIOU, C.;.PAPANASTASIOU, E. (1997). Factors that influence students to become teachers. Educational Research and Evaluation (an International Journal on theory and Practice); Vol. 3; núm. 4, pp. 305-16 
Perfil de la educación en México (1999). Secretaría de Educación Pública. 2ª . Edición. México.

RAMOS J, R.S. y ESQUIVEL, L (2002). Motivación hacia el estudio, expectativas y características familiares de mujeres inscritas en una licenciatura en Psicología. Educación y ciencia. Nueva ëpoca, Vol. 6, núm. 11 (25). Enero-junio. pp. 61-73

SÁNCHEZ, P y VALDÉS, A. (2003). Teoría y práctica de la orientación en la escuela México: Editorial Manual Moderno.

SORIA N, O. (2000). Motivos para oferta y demanda de posgrado. En Moreno, B.,

Soria N., Garibay B., Sánchez P., ARRedondo G., Sánchez E. y díaz B. C. (2000). Problemática de los Posgrados en Educación en México: Hacia la consolidación en el siglo XXI. Universidad Autónoma del Carmen. Cuadernos de Investigación 5.

Sprinthall N. A., SpRinthall R. C. y OJA S. N. (1996). Psicología de la Educación. España: Mc Graw Hill

WoOlfolk, A.E. (1996). Psicología educativa.(2ª . Ed.). México: PrenticeHall Hispanoamericana

WU, WEI (2000). Motives of Chinese Students to Choose Journalism Careers. Journalism and Mass Communication Educator; Vol. 55, núm. 1, pp. 53-65 


\title{
Contactar
}

Revista lberoamericana de Educación

\author{
Principal OEI
}

